\section{Experimental design and statistical analysis}

Experiments: Design and Analysis. Second edition. By J. A. John and M. H. Quenouille. Pp. 296. (Charles Griffin: London and High Wycombe. 1977.) $£ 12$.

The Design and Analysis of Experiment by Quenouille, first published in 1953, was an early text which emphasised the practical aspects of experimental design and statistical analysis. The second edition, which appears under the joint authorship of J. A. John and Quenouille, who died at an early stage in its preparation, preserves the spirit of the original, with detailed coverage of the more widely used designs fully illustrated by numerical examples from either agricultural or animal experiments.

Despite the high standard of the original, the changes incorporated in the new edition are welcome; the grouping of chapters into sections has been abandoned and some re-arrangement has allowed the inclusion of two new chapters-one on fractional replication and the other on response surface methods-without increasing their total number. The contents have been updated with advances made since first publication, and strengthened with further elaboration of specific designs. The bibliography has been revised and expanded.

The first four chapters discuss randomised blocks, Latin squares, and factorial and split-plot designs. Techniques for missing-values, originally confined to a separate chapter, have been inserted at the appropriate places. Discussion of missing-values in randomised blocks is particularly extensive, although it is strange that two missing-values in such designs should be estimated by iteration rather than directly equating the appropriate residuals to zero.

The next four chapters illustrate the main modifications of full factorials through confounding and partial replication. The new chapter 9, on response surface methods, provides an excellent account of first- and second-order designs, including rotatability; examination of the fitted response surface is particularly well illustrated.

Chapter 10, on incomplete block designs, includes recent work on resolvable and cyclic designs and a brief introduction to partially-balanced incomplete blocks. The three chapters (11, 12 and 13) on designs for estimating residual effects in long-term experiments, planning of groups of ex- periments and combination of results, still constitute one of the few adequate expositions of these important topics. The final chapter describes the scaling of data, and the book ends with tables of $t, F, \chi^{2}$, studentised range and random permutations, a bibliography and a good index.

This book is a valuable guide to the understanding of experimental design: its discussion of real data and the

\section{Population differentiation and gene-flow}

Geographic Variation, Speciation, and Clines. By John A. Endler. Pp. $\mathrm{ix}+246$. (Princeton University: Princeton, New Jersey, 1977.) Hardback $£ 12$ : paperback $£ 5.45$.

Charles Darwin, with his knack for picking a good problem, wrote: "Those forms which possess in some considerable degree the character of species, but .... are so closely linked ... by intermediate gradations, that naturalists do not like to rank them as distinct species, are in several respects the most important to us." Endler's book reviews the diverse and scattered literature on clines-that is, the study of population differentiation in the absence of barriers to gene-flow-that has accumulated since Darwin.

It directs itself to two fundamental questions. First, it asks whether sharp geographical differentiation can evolve across a spatially or genetically continuous series of populations; or whether gene-flow can prevent spatial differentiation. The answer is rather clear. Subspecific and even specific differentiation (parapatric speciation) in the absence of barriers to gene-flow is as common and important as divergence with strong barriers, whatever our introductory textbooks might say.

Second, it asks about the shape of clines; for example, whether stepped clines require stepped environments and whether there is always a unique one-to-one correspondence between local steepening and environmental change. Endler explores the rich possibilities in considerable detail, using analytical and computer models, as well as laboratory populations. The fairest summary is that "it depends". Chapter 4, in particular, shows how conclusions based on classical singlelocus models can be much influenced by the effects of co-adapted modifiers.

Whereas chapters 2-4 consist of rather detailed population genetics, the last two chapters, which they underpin, are of more general interest. Chapter 5 elaborates the earlier con- absence of mathematical and statistical models should appeal to the experimenter. Its comprehensive coverage of the simpler designs and the tabular summaries of their properties provide a useful reference for the statistician.

A. L. Johnson

A. L. Johnson is a statistician in the $M R C$ Statistical Research and Services Unit at University College Hospital Medical School, London, UK.

clusion that parapatric speciation is probably common. Indeed, present-day patterns of distribution resulting from parapatric speciation do not differ significantly from those generated by allopatric speciation and secondary contact. Even the familiar explanations for present-day distribution patterns of Amazonian and African forest birds, butterflies and other organisms, based on Pleistocene refugia within forest remnants and subsequent secondary contact, look shaky in the light of Endler's reanalysis in chapter 6 . These distributions can equally well be explained by the presence of some kind of selection gradient, which may be sufficient to maintain geographical differentiation regardless of whether or not the forests have been fragmented.

The book summarises a great deal of empirical data on clines of all kinds, and makes a brave attempt to match theory with data wherever possible. Efforts to assign numbers to key parameters-for example, rates and distances of gene-flow and cline width (the distance over which gene-frequencies change)-are particularly commendable. Many of the estimates may be no better than an order of magnitude, but they can nevertheless constrain the biologically feasible solutions to general models in a dramatic and cautionary way. Nevertheless, I was left with the distinct impression that theory can now generate so many alternative explanations for apparently simple phenomena that distinguishing hypotheses would in many cases be a major undertaking, if not downright impossible.

Before I read the book, I knew very little about clines, other than that they were interesting. When I was reading it, there were times when 1 felt that $I$ was finding out more than 1 really wanted to know; that somehow, Darwin's clear, simple problem had been lost in the toolkit of modern population genetics. But by the time I finished it, I was more convinced than ever that clines are intriguing. For any biologist brought up in the simple belief that allopatric speciation is all, this book is essential reading. John $H$. Lawton

John Lawton is Lecturer in Ecology at the University of York, UK. 